\title{
Cuidado em saúde mental: significados e estratégias para os familiares
}

\author{
Mental health care: meanings and strategies for families \\ Atención de salud mental: significados y estrategias para las familias
}

\section{RESUMO}

Objetivo: Compreender os significados e as estratégias elaboradas pelos familiares de pacientes em acompanhamento de saúde mental. Método: A pesquisa é do tipo exploratório-descritiva, com abordagem qualitativa, que ocorreu em um Centro de Atenção Psicossocial (CAPS I), no Nordeste. A pesquisa foi composta por 20 familiares de pacientes em sofrimento psíquico, mediante acessibilidade e conveniência. A técnica de coleta de dados foi a de Grupo Focal (GF), mediante questões norteadoras do roteiro, com perguntas reflexivas, o GF foi guiado. Fez-se o uso da Análise de Bardin. A pesquisa seguiu os preceitos éticos, sob o CAAE: 22372113.0.0000.5182. Resultados: Foram construídas duas categorias: I- Significado do Cuidado e II - Estratégias de enfrentamento para as adversidades. Conclusão: 0 estudo alcançou o seu objetivo inicial e em suas categorias, percebeu-se a complexidade do cuidado em saúde mental e a necessidade de novas estratégias junto à família dos pacientes.

DESCRITORES: Cuidado; Sobrecarga; Saúde mental; Estratégias de Saúde

\section{ABSTRACT}

Objective: To understand the meanings and strategies developed by the families of patients in mental health monitoring. Method: The research is exploratory-descriptive, with a qualitative approach, which took place in a Psychosocial Care Center (CAPS I), in the Northeast. The research consisted of 20 family members of patients in psychological distress, through accessibility and convenience. The data collection technique was the Focus Group (FG), through guiding questions of the script, with reflective questions, the FG was guided. The Bardin Analysis was used. The research followed the ethical precepts, under the CAAE: 22372113.0.0000.5182. Results: Two categories were built: I- Meaning of Care and II - Coping strategies for adversities. Conclusion: The study reached its initial objective and in its categories, the complexity of mental health care and the need for new strategies with the patients' families were perceived.

DESCRIPTORS: Care; Overload; Mental health; Health Strategies.

\section{RESUMEN}

Objetivo: Comprender los significados y estrategias que desarrollan las familias de los pacientes en el seguimiento de la salud mental. Método: La investigación es exploratoria-descriptiva, con abordaje cualitativo, que se llevó a cabo en un Centro de Atención Psicosocial (CAPS I), en el Nordeste. La investigación consistió en 20 familiares de pacientes en distrés psicológico, a través de accesibilidad y conveniencia. La técnica de recolección de datos fue el Focus Group (FG), a través de preguntas orientadoras del guión, con preguntas reflexivas, se guió el FG. Se utilizó el análisis de Bardina. La investigación siguió los preceptos éticos, bajo el CAAE: 22372113.0.0000.5182. Resultados: Se construyeron dos categorías: I- Significado del cuidado y II - Estrategias de afrontamiento de las adversidades. Conclusión: El estudio alcanzó su objetivo inicial y en sus categorías se percibió la complejidad de la atención en salud mental y la necesidad de nuevas estrategias con las familias de los pacientes.

DESCRIPTORES: Atención; Sobrecarga; Salud mental; Estrategias de salud

RECEBIDO EM: 28/06/2021 APROVADO EM: 18/01/2022

\section{Aline Pereira da Silva}

Enfermeira. Pós-graduada em obstetrícia e neonatologia. Universidade Federal de Campina Grande.

ORCID: 0000-0001-8185-9471.

\section{Perla Figueredo Carreiro Soares}

Enfermeira. Mestre em Neurociência Cognitiva e Comportamento. Pós-graduada em enfermagem obstétrica. Chefe do Núcleo de Serviços Diagnósticos/SES/PB.

ORCID: 0000-0002-0407-685X 


\section{Erlânia Souza Costa}

Enfermeira. Pós-graduada em obstetrícia e neonatologia. Faculdade de Enfermagem Nova Esperança.

ORCID: 0000-0002-5018-0404.

\section{Lúcia Gomes de Souza Silva}

Enfermeira. Faculdade Maurício de Nassau. Pós-graduada em Urgência/Emergência e Unidade de Terapia Intensiva.

ORCID: 0000-0001-6998-435X.

\section{Rogéria Gomes da Silva}

Enfermeira. Pós-graduada em Gestão em Saúde, Gestão da Clínica nas regiões de Saúde e Docência do Ensino Superior. Chefe do Núcleo de Atenção Ambulatorial/SES/PB.

ORCID: 0000-0001-7075-1369.

\section{Luanna Silva Braga}

Enfermeira. Mestre em Enfermagem pelo Programa de Pós-Graduação em Enfermagem da Universidade Federal da Paraíba (PPGENF/UFPB).

ORCID: 0000-0002-0093-0406.

\section{INTRODUÇÃO}

A Reforma Psiquiátrica caracteriza-se como um movimento histórico de caráter político, social e econômico, que se desenvolveu paralelamente à Reforma Sanitária e tem como finalidade a substituição progressiva dos manicômios por outros serviços junto à comunidade, deslocando a intervenção terapêutica para o contexto social das pessoas, desencadeando um processo de transformação na organização dos sistemas de saúde mental (1).

Para efetividade desse processo, é indispensável que se desenvolvam estratégias para que se possa trabalhar com pessoas em sofrimento psíquico egressas de internações de longa permanência, de forma que possam ser inseridas e integradas na própria família e comunidade, considerando que a própria condição de "doente mental", por si só, acarreta ao indivíduo acometido por sofrimento psíquico e seus familiares algumas limitações, impedimento e situações que podem alterar a relação da pessoa com o trabalho, com os demais membros da família, amigos e parceiros, bem como abalar sua identidade(1).

Em 6 de abril de 2001, o Governo Federal promulga a Lei n. ${ }^{\circ} 10.216$ que dispõe sobre a proteção e os direitos das pessoas em sofrimento psíquico e redireciona o modelo assistencial em saúde mental. A lei redireciona o modelo da assistência psiquiátrica, regulamenta cuidado especial com a clientela internada por longos anos e prevê a possibilidade de punição para a internação voluntária arbitrária ou desnecessária(2).

A reforma psiquiátrica brasileira é respaldada pela Lei $\mathrm{n}^{\circ} 10.216$ e por inúmeras portarias inseridas pelo Ministério da Saúde (MS) com o intuito de prestar uma assistência de qualidade ao portador de sofrimento psíquico e seus familiares. $\mathrm{O}$ projeto em extensão constitui-se pelo modelo comunitário com seus diversos dispositivos institucionais: os Centros de Atenção Psicossocial (CAPS) como ordenadores da rede; o programa De Volta para Casa, que reinsere usuários de longa permanência internados em hospitais psiquiátricos na sociedade; os leitos psiquiátricos em hospital geral; a construção de uma política pública intersetorial para álcool e drogas, e também para crianças e adolescentes; a articulação com a rede básica de saúde; e a consequente redução dos leitos nos hospitais psiquiátri$\cos (3)$.

O parágrafo anterior, reflete justamente as estratégias utilizadas atualmente diante da política de saúde mental, que tem dentre os seus objetivos, viabilizar o cuidado com diferentes formas de prestar assistência em saúde para o paciente e sua família. O novo modelo de assistência ao sofrimento mental está centrado na reintegração dessas pessoas na família e comunidade, são tarefas às quais o SUS vem se dedicando com especial empenho.

Diante disso, questionou-se: Como se dá o cuidado em saúde mental na perspectiva dos significados e estratégias elaboradas pelos familiares. Tem-se por objetivo: compreender os significados e estratégias elaboradas pelos familiares de pacientes em acompanhamento de saúde mental.

\section{MÉTODO}

A pesquisa é do tipo exploratório-descritivo, com abordagem qualitativa. Este estudo ocorreu em um Centro de Atenção Psicossocial (CAPS I), no município de um estado nordestino, no ano de 2014, no primeiro trimestre. A população total do CAPS é de 78 usuários, porém, apenas 40 estão ativos na unidade de saúde. Destes 78 usuários, somente 20 frequentam o serviço todos os dias. Desse modo, a amostra desta pesquisa, foi composta por 20 familiares de pacientes em sofrimento psíquico, mediante acessibilidade e conveniência.

Continuamente, a pesquisa teve como critérios de inclusão: ser familiar do usuário, e este precisa estar ativo no serviço de saúde do CAPS, aceitar participar da pesquisa e assinar o termo de consentimento livre e esclarecido (TCLE). Sendo assim, aqueles que não correspondiam aos critérios de inclusão, foram excluídos. A técnica de coleta de dados foi a de Grupo Focal 
(GF), proporcionando interação, construção de problemáticas e transformação da realidade dos participantes(4). No GF acontecem alguns encontros em grupo, no qual os participantes deixam suas opiniões e reflexões sobre o tema em estudo(4). O GF teve duração de 90 minutos, para que as falas fossem exploradas da melhor forma possível. Foram indagadas quatro questões abertas, guiadas por um roteiro, além de um questionário inicial, que traçava o perfil dos participantes, neste questionários, as questôes eram abertas e fechadas, versando perguntas de caracterização dos participantes da pesquisa, tais como: sexo, idade, tempo de atuação no setor de pesquisa entre outras perguntas.

Mediante questões norteadoras do roteiro, com perguntas reflexivas, o GF foi guiado. Todo o conteúdo foi gravado por um aparelho eletrônico na modalidade MP3, além disso, fez-se o uso da Análise Temática de conteúdo de $\operatorname{Bardin}(5)$. A pesquisa seguiu os preceitos éticos, sob o CAAE: 22372113.0.0000.5182, em Março, ano de 2014.

\section{RESULTADOS}

Diante das etapas de análise, a amostra se caracterizou da seguinte forma: do total de 20 participantes, 15 estavam na faixa etária de 40 a 60 anos, e os demais entre 25 a 40 anos. Os familiares eram: tias, genitoras e irmãs dos pacientes em sofrimento psíquico. Foram construídas duas categorias: I- Significado do Cuidado e II - Estratégias de enfrentamento para as adversidades.

\section{Categoria I - Significado do Cuidado}

Quando se cuida, pressupóe-se gerar no ser cuidado um sentimento de retribuição, carinho, compreensão, ou seja, um sentimento a ser oferecido buscando-se vários outros ocultos. Por isso, quando se oferece um sentimento, resgata-se e aprimora-se o sentimento dos outros, com a finalidade recíproca de que isso perpetue por um longo tempo entre um e o outro.

Os participantes entrevistados descrevem por meio de suas falas uma perfeita compreensão acerca do cuidado na sua con-

cepção:

“O cuidado é

direto! Às vezes

estou dormindo e

acordo para saber

como ele está,

porque a pessoa

fica preocupada,

fica pensando será

que ele tá bem?,

aí vou olhar como

ele está. Quando

passa cinco ou dez

minutos que ele

não chega da escola

já fica preocupada

pensando que foi

atropelado aí acabo

deixando meus

afazeres e vou atrás

dele". (Participante

12)
"Cuidado é você ter interesse na vida do outro, se preocupar, dar carinho, atenção é saber apoiar nos momentos difíceis como também ensinar o certo e o errado, até porque cuidar também é educar". (Participante 7)

"Cuidado é você fazer o bem, até porque a gente só cuida e se preocupa com quem a gente ama". (Participante 13)

O desenvolvimento da capacidade de cuidar é essencial para o desenvolvimento do ser. Os comportamentos básicos de cuidar são: compaixão, competência, confiança, consciência e compromisso.

De acordo com os relatos dos participantes da pesquisa, os familiares cuidadores consomem a maior parte de seu tempo dedicando-se atendendo às necessidades do familiar em sofrimento psíquico de forma a colocar suas necessidades e desejos em segundo plano. Os participantes abordam as seguintes informações acerca do que significa cuidar de um familiar em sofrimento mental:

"Cuidar de um filho especial é um trabalho dobrado". (Participante 1)

"Cuidar de um filho especial exige um maior tempo de dedicação". (Participante 7)

"O cuidado é direto! Às vezes estou dormindo e acordo para saber como ele está, porque a pessoa fica preocupada, fica pensando será que ele tá bem?, ai vou olhar como ele está. 2uando passa cinco ou dez minutos que ele não chega da escola já fica preocupada pensando que foi atropelado ai acabo deixando meus afazeres e vou atrás dele". (Participante 12)

Nestes relatos fica evidente que cuidar de um familiar sofrimento psíquico requer 
uma responsabilidade maior. Ao assumir tal função, o familiar cuidador passa a desempenhar diretamente o papel de observador, avaliador, determinando as ações e decisões a serem tomadas, surgindo, assim, o estresse e a ansiedade pela transformação das situações vivenciadas. Este familiar passa a viver um cotidiano impregnado de preocupação. Nesse sentido, os transtornos mentais são vividos de maneira coletiva, pelo grupo familiar como um todo. Quando um membro familiar adoece, toda a sua rede de relações se altera, sendo a família, na maioria das vezes, o grupo primário de inserção de um indivíduo.

\section{No confronto com}

a doença as pessoas

ficam fragilizadas

emocionalmente,

\section{muitas vezes}

recorre a uma ajuda

espiritual, seja

na hora da dor,

da desesperança,

e na busca de

significados para os

eventos de sua vida
Categoriall-Estratégiasdeenfrentamento para as adversidades

Ao analisar o material coletado é perceptível a dificuldade que os familiares/ cuidadores encontram para traçar estratégias frente às adversidades diárias. Todos os participantes referiram a fé como um forte aliado para superar as dificuldades diárias. As falas a seguir enfatizam essa questão:

[...] dificuldade todo mundo tem, independente de ter um parente especial ou não, mas quando a gente crer em Deus todos os problemas e as dificuldades vão embora. (Participante 6)

Quando tenho muitos problemas vou à igreja, Deus é tão maravilhoso que quando a gente vê não existe nenbuma dificuldade. (Participante 7)

Só Deus para nos ajudar nos momentos de dificuldades. (Participante 19)

No confronto com a doença as pessoas ficam fragilizadas emocionalmente, muitas vezes recorre a uma ajuda espiritual, seja na hora da dor, da desesperança, e na busca de significados para os eventos de sua vida. A doença pode tornar-se uma situação de aproximação com o divino na tentativa de salvação ou de resolução dos problemas. Quanto mais apegada aos aspectos espirituais, mais a família identifica recursos e mantém sua energia para levar a situação estressante adiante. A religião ou religiosidade não tem poder de resolver a situação instantaneamente, mas sim, de ir renovando as energias para que a família vá identificando recursos e aprendendo a lidar com as situações.

A família utiliza recursos de ordem religiosa para entender a doença e lidar com ela. Assim, crenças religiosas e valores são utilizados pela família para ajudar a dar sentido ao evento de doença do familiar em sofrimento psíquico.
Os resultados revelam várias estratégias e serviços substitutivos para os pacientes e familiares estarem inseridos. As residências terapêuticas ou simplesmente moradias, são locais destinados a atender aos transtornos mentais, são casas de localização urbana, tendo como finalidade atender as necessidades de moradia de pessoas com sofrimento mental grave e persistente, egressas de hospitais psiquiátricos, ou não. Embora as residências terapêuticas se configurem como sendo um equipamento de saúde, devem ser capazes de garantir o direito à moradia aos indivíduos com transtorno mental como também auxiliar o morador em seu processo de reintegração na comunidade(6).

O Programa de Volta para Casa configura-se como uma estratégia potencializadora de emancipação de pessoas com sofrimento psíquico. Criada pela lei federal 10.708 e sancionada em 2003, esse programa é uma parceria do Ministério da Saúde e da Caixa Econômica Federal com os municípios conveniados, visa promover e facilitar o processo de reabilitação e inserção social de pessoas acometidas de transtornos mentais, que tenham um histórico de vida com 02 (dois) ou mais anos de internações nos hospitais psiquiátricos(7).

O Centro de Atenção Psicossocial (CAPS) é apresentado pelo Ministério da Saúde como dispositivo prioritário de atenção à saúde mental. Pretende-se com sua implantação e operação, inovar a assistência na perspectiva da promoção da saúde e uma ruptura com o modelo assistencial hegemônico. O CAPS, como organizador do sistema de atenção em saúde mental, tem a responsabilidade social não só de dar conta de substituir o modelo manicomial, como proporcionar estratégias e programas de promoção da saúde. A começar pela sua articulação com a comunidade, visando sua participação efetiva e concreta na eleição de prioridades, na tomada de decisóes e na elaboração e desenvolvimento de estratégias para alcançar melhor nível de saúde(6).

A portaria 3.088, de 2011, do Ministério da saúde, define as seguintes modalidades de CAPS: CAPS I, CAPS II, CAPS 
III, CAPS Ad, CAPS AD, CAPS AD III e CAPS i, por ordem crescente de porte, complexidade e abrangência populacional. Cumprem a mesma função no atendimento público em saúde mental. Responsabiliza-se, sob coordenação do gestor local, pela organização da demanda e da rede de cuidado em saúde mental no âmbito do seu território. O ambiente deve estar capacitado para realizar atendimento a clientes com transtornos mentais severos e persistentes, em regime de tratamento intensivo, semi-intensivo e não intensivo. A portaria também determina que o CAPS supervisione e capacite as equipes de atenção básica, serviços e programas de saúde mental no seu âmbito(8).

Os CAPS configuram-se como serviços comunitários ambulatoriais e regionalizados, nos quais os pacientes deverão receber consultas médicas, atendimentos terapêuticos individuais e/ou grupais, podendo participar de ateliês abertos, de atividades lúdicas e recreativas promovidas pelos profissionais do serviço, de maneira mais ou menos intensa e articulada em torno de um projeto terapêutico individualizado voltado para o tratamento e reabilitação psicossocial, devendo também haver iniciativas extensivas aos familiares e às questões de ordem social presentes no cotidiano dos usuários(9).

Esse novo modelo, que ultrapassa a ideia de simplesmente promover a desospitalização, acarretou no cotidiano inúmeros problemas. Na trajetória temporária para uma nova organização assistencial, as primeiras ações visíveis se caracterizaram pela diminuição das internações em hospitais psiquiátricos e pela criação de recursos assistenciais de caráter extra-hospitalar(10).

Os profissionais dos serviços extra-hospitalares, como o CAPS e as residências terapêuticas seguem os princípios da integralidade, auxiliando o usuário em todas as áreas do ser humano: biopsicossocial e espiritual, não fragmentando o cuidado. Tornando o cuidado humanizado, existindo vínculos entre equipe e usuário e havendo a responsabilização de ambos pelo cuidado ofertado. Os profissionais do serviço no decorrer das atividades procuram abrir espa- ços para conversas individuais, ou mesmo com o grupo, na intenção de aliviar ansiedades, medos e debater situações específicas que são vivenciadas pelos usuários(11).

Para atuar junto às famílias de sofrimento psíquico, é importante que o enfermeiro conheça o cotidiano e a estrutura familiar, suas crenças, valores e conhecimento sobre a doença, para que, a partir daí, possa programar sua assistência necessária, conforme a necessidade desses familiares(12).

Este vínculo estabelecido entre o profissional com o usuário é visto como facilitador no tratamento, havendo fidelidade entre ambas as partes. Assim, o cuidado em saúde mental ganha materialidade na atitude dos profissionais do CAPS, na sua relação com os usuários, tendo a integralidade como foco de intervenção, ou seja, é um local de encontro, diálogo entre pessoas com necessidades, desejos, histórias e conhecimentos específicos. O cuidado proposto por esses profissionais está além dos usuários, oferecendo à família suporte, tendo em vista que a família é parte fundamental para a evolução satisfatória do usuário(11).

No entanto, essas medidas implicaram intensificação das exigências de comprometimento das famílias dos indivíduos com sofrimento psíquico. Transformado de modo significativo à trajetória de sua participação no processo assistencial. No conjunto, novas demandas foram impostas, sem que tenham sido instrumentalizadas para atendê-las, acarretando-lhes sensação de desamparo(10).

A família torna-se responsável por atender às suas necessidades básicas, como coordenar suas atividades diárias; administrar sua medicação; acompanhá-los aos serviços de saúde; lidar com suas condutas problemáticas e episódios de crise; fornecer-lhes suporte social; arcar com seus gastos; e superar as dificuldades dessas tarefas e seu impacto na vida social e profissional do fa$\operatorname{miliar}(10)$.

O transtorno mental grave, além de causar mal físico e psíquico ao paciente, acarreta mudanças no cotidiano das pessoas que o cercam. Observa-se que a participação da família na assistência em saúde mental tem mostrado convergência. Há a necessidade
Este vínculo

estabelecido entre

o profissional com

o usuário é visto

como facilitador no

tratamento, havendo

fidelidade entre

ambas as partes.

Assim, o cuidado

em saúde mental

ganha materialidade

na atitude dos

profissionais do

CAPS, na sua

relação com os

usuários, tendo a

integralidade como

foco de intervenção,

ou seja, é um local

de encontro, diálogo

entre pessoas com

necessidades,

desejos, histórias

e conhecimentos

específicos 


\section{artigo}

de prover a família do indivíduo acometido pelo sofrimento psíquico de informações e orientações para que, dessa forma, a família possa colaborar como agente ressocializador e facilitador do doente na família e na comunidade(14).

Cuidar de um familiar com sofrimento psíquico impõe aos familiares situações agregadas aos sintomas da doença, com as quais nem sempre está disposto a suportar. Estas situações incluem comportamentos de descontrole do familiar doente, que se torna mais agressivo e inquieto, causando um clima de intranquilidade que foge aos padrões até então conhecidos pelos que, com ele, convivem e que afetam toda a estrutura familiar(12).

O familiar cuidador pode desenvolver ansiedade por não saber como lidar com alguns comportamentos apresentados pelo sofrimento psíquico como, por exemplo, o silêncio exagerado, fala contínua e desordenada ou com a imprevisibilidade em suas ações, e a percepção da vida cada vez mais "empobrecida" do paciente. Muitos não se conformam em ver um familiar, que até então era brilhante, cheio de projetos de vida e socialmente bem integrado, transformar-se numa pessoa comprometida, dependente, desprotegida e tomada por limitações de toda natureza(12).

Diante desse novo modo de assistência, a família é vista como aliada no que se refere ao processo de cuidado à pessoa com sofrimento psíquico, porém essas famílias necessitam que os profissionais de saúde ofereçam suporte para que possam manter o núcleo familiar saudável, já que o conviver com um familiar em sofrimento psíquico impõe mudanças no cotidiano da família como: alteraçôes na rotina do sono, prejuízos ao trabalho e à situação financeira, a necessidade de cuidar do familiar quanto à administração dos medicamentos e higiene corporal e sobrecarga física e psicológica(14). Sendo assim, a Atenção Primária à Saúde (APS) também tem sua responsabilidade, buscando o cuidado longitudinal, pois é uma ferramenta para ampliar a assistência oferecida ao paciente em sofrimento mental, quebrando desafios e potencializando a saúde (15).
O estudo alcançou o seu objetivo inicial e em suas categorias, percebeu-se o significado de cuidado dentro do GF, no qual referiu-se a dedicar parte de sua vida ao outro, promovendo o bem estar físico, emocional e psicológico ao ser cuidado. Em outra categoria desta pesquisa, foram apontadas novas estratégias específicas para o cuidado ao familiar em sofrimento psíquico. Porém, diante dos problemas enfrentados diariamente, o GF demonstrou que a fé tem sido a principal estratégia para vencer junto ao paciente no cuidado em saúde mental.

Para tanto, torna-se essencial que se reconheça o tipo de famílias existentes, se fortaleça a rede de atenção psicossocial da região, e que os vínculos entre familiares e usuários sejam alicerçados numa relação de respeito, onde a singularidade e individualidade de todos sejam preservadas, de forma que os familiares/cuidadores não se sintam sobrecarregados, e os portadores de sofrimento psíquico possam resgatar sua cidadania e autonomia.

\section{REFERÊNCIAS}

\section{CONCLUSÃo}

1- Mattioni FC. et al. Refletindo acerca do cuidado ao sujeito em sofrimento psíquico: o processo de inclusão aliado a desinstitucionalização. Ciências, Cuidado e Saúde, v. 7, 2013.

2-Berlinck MT, Magtaz AC, Teixeira MA. Reforma Psiquiátrica Brasileira: perspectivas e problemas. Revista Latinoamericana de Psicopatologia Fundamental, São Paulo, v. 11, n. 1, p. 21-27, mar. 2008.

3- Amarante $P$, Diaz SF. Os movimentos sociais na reforma psiquiátrica. Caderno Brasileiro de Saúde Mental. Rio de Janeiro, v. 4, n. 8, p. 83-95, jan./jun. 2012.

4- Backes DS. et al. Grupo focal como técnica de coleta e análise de dados em pesquisas qualitativas. 0 Mundo da Saúde, v. 35, n. 4, p. 438-442, 2011.

5- Bardin, L. Análise de conteúdo. 2006.

6- Brasil. Ministério da Saúde. Secretaria de Atenção à Saúde. DAPE: Coordenação Geral de Saúde Mental. Reforma psiquiátrica e políticas de saúde mental no Brasil. Documento apresentado à Conferência Regional de Reforma dos Serviços de Saúde Mental: 15 anos depois de Caracas. OPAS. Brasília, novembro de 2005.

7- Freire FH. Silva VN. Oliveira MFO. "Programa de volta pra casa" no campo da saúde coletiva e saúde mental: estratégias de desinstitucionalização e reabilitação psicossocial. Revista Científica da Escola de Saúde, Natal, v. 2, n. 1, p. 51-62, 2012.
8- Portaria GM 3088, de 23 de dezembro de 2011.

9- Tavares CMM, Souza MT, Rodrigues SP. Participação da comunidade nos Centros de Atenção Psicossocial - CAPS. Revista de Pesquisa: cuidado é fundamentaL. v. 2, n. 2, p. 936-946, abr./jun. 2010.

10- Cavalheri SC. Transformações do modelo assistencial em saúde mental e seu impacto na família. Revista Brasileira de Enfermagem, Brasilia, v. 63, n. 1, p. 51-57, jan./fev. 2010.

11- Mielke FB. et al. 0 cuidado em saúde mental no CAPS no entendimento dos profissionais. Ciência \& Saúde Coletiva, Rio Grande de Sul, v. 14, n. 1, p. 159-164, 2009.

12- Sant'ana MM. et al. O significado de ser familiar cuidador do portador de transtorno mental. Texto Contexto Enfermagem, Florianópolis, v. 20, n. 1, p. 50, jan./mar. 2011.

13- Clasen BN, Kantorski LP. Schwartz E. Sofrimento psíquico e família. Ciência, Cuidado e Saúde, Rio Grande do Sul, v. 7, 2013.

14- Bessa JB, Waidman MAP. Família da pessoa com transtorno mental e suas necessidades na assistência psiquiátrica. Texto Contexto Enferm. Florianópolis, v. 22, n.1, p. 61-70, jan./mar. 2013.

15- Nascimento EPL, Vizelli C, Mariano RCZ, Romano TCR. Ferramentas utilizadas no cuidado continuado em saúde mental no contexto da COVID-19: uma revisão integrativa. saúde coletiva, 2021; (11) N.62. 